\title{
Food Hygiene and Quality Control in Food Processing
}

\author{
Dehong Du
}

College of food science and engineering, Qingdao Agricultural University, Qingdao, 266109, China

hunter2011@foxmail.com

Keywords: Food processing, Hygiene and quality control, Market reputation

\begin{abstract}
Food safety is crucial to guarantee human life health safety and promote social stability and social stability. However, as various kinds of food increase in the market, food quality and safety accidents take place frequently, and food processing and production are faced with severe challenges. Thus, hygiene and quality control in food processing is particularly important. Meanwhile, it is the key to improving business market reputation and maintaining social harmony and stability. This paper will analyze hygiene and quality control methods in food processing to show the importance of food quality control.
\end{abstract}

\section{Introduction}

With social development and progress, food has changed to consumption goods from original plantation or breeding, processing and circulation and become indispensable commodities in consumers' life. Food has experienced a long process from food to commodity. Every link in food processing and production concerns final food quality, and food quality and safety accident will occur in each link. Relevant survey shows most safety and quality problems appear in food processing and production (accounting for above 80\%). 5\% food quality problems happen in the quality of raw materials; $10 \%$ of safety problems appear at the terminal; $4 \%$ of safety problems appear in food circulation. Thus, quality control in food production and processing link is pivotal.

\section{Quality and safety problems in food processing process}

Current situation of food production and processing in China. Food industry in China has experience long-term development and evolution. Food industry has been very developed and food varieties are very rich. Hence, food industry becomes a pillar industry in China. The development of food industry is confronted with numerous problems and food challenges. Gross value of food industry is a key index to measure development level of food industry. The proportion of food industry is $1.7: 2 \sim 3: 1$ in developed countries, while the proportion of total output value of food industry is $0.34: 2$ in China. There is still a gap with developed countries. Besides, food industry accounts for $10 \%$ of total food consumption in China, while this figure is $70 \%$ in developed countries and 50\% in other developing countries. In 2005, total value of food industry in China was only 190 million, less than $1 / 3$ in the world. It thus can be seen that China still has a significant gap with developed countries in food industry, and overall development level falls behind.

Even if the reserve of grain, vegetable, fruit and meat is very abundant, and they rank top in the world, many shortcomings exist in food processing and production. Low processing degree and semi-finished products still account for a large proportion, and the number of finished products is small. In food deep processing, the amount of deep processing is very limited, and accounts for only $7 \%$ of total food deep processing, while that figure is above $60 \%$ in developed countries. In one word, seeing from current food processing level, many defects exist and industrial development is faced with numerous problems. To narrow the gap with developed countries and improve food processing quality, it is required to combine development status, formulate and optimize development strategy.

Problems in food processing. Food industry is most closely related to people's life. China is a multinational state, and regional differences are significant. In particular, significant differences exist in various regions in terms of dietary habits and consumption level. Regional differences are more 
significant ii many regions and especially seasonal food consumption market. China's food industry includes many categories. This is also an important reason why separation starts to appear in food market. This also results in the rise in the number of enterprises. These enterprises are confronted with fierce market competition and have been in development edge. Besides, their scale is narrow and enterprises are scattered. For instance, as a monopolizing industry, there are numerous famous manufacturers in liquor industry, but they are small distilleries and faced with the crisis of merger. Tobacco industry is also a nationwide leading enterprise, but it tobacco industry in fierce market competitions. Thus, the pattern where famous enterprises, medium and small enterprises coexist ${ }^{[1]}$.

The number of the enterprises is many, and the scale is small. Manufacturing techniques of many enterprises fall behind; resource consumption is serious; economic benefit and social benefit are very low. For example, average annual output of China's beer brewing enterprises is 48000t; average annual production scale of wind enterprises is small, and the annual output is only 1200t; average annual output of beverage factories is 1500t. All these enterprises own numerous production sites. Even if small and medium enterprises have sought new development opportunities continuously in recent years and expanded the scale, they are still in the adverse situation in food processing and production.

Research and development ability of enterprises is poor, and technical innovation is weak. Based on the above discussion, we can know China's food industry development is very slow. Food processing enterprises have shortcomings in many production links. Insufficient research and development ability and weak technical innovation are important factors hindering enterprise development. Relevant survey shows the proportion of research and development funds inputted by food processing enterprises accounts for $1.7 \%$ of GDP, while this figure is $4 \% \sim 6 \%$ in developed countries. The difference is significant. The overall level of technological achievement transformation is low. Scientific and technical cost invested by China in food processing and production is very limited. China puts particular emphasis on scientific research on food and does not pay much attention to quality control. Food processing and production innovation mainly depend on scientific research input of enterprises. But, the funds of enterprises are limited; obstruction exists in capital turnover; the investment proportion of research and development expenditure is low; technical personnel are in self-development stage, and it is difficult to find out breakthroughs technically. Thus, the deficiency of management and scientific research strength will result in poor product quality and low grade.

Quality safety problem in food processing link is severe. Food inspection data of a region in 2010, 2011 and 2012 indicate many quality problems exist in food production and processing links. For example, label is unqualified; microorganism exceeds standards or there are toxic and harmful substances. Unqualified label means among the inspected food, there is label mistake, standard code mistake, quality grade mistake, no net content or no marked ingredient or additive. Besides, labeling operation is not standard enough. Specific food name or production code is not marked, so accurate attribute of food cannot be really reflected. Unqualified component content is reflected in the following: raw materials are adulterated; important components do not comply with norms. For instance, moisture or additive exceeds standards; the content of brightening agent, sweetening agent or antiseptic substance is below standard. Unqualified microorganism means the content of escherichia coli, saccharomycetes and mould exceeds the standard. These food safety problems will give rise to threats to human health if they are not valued ${ }^{[2]}$.

There are many reasons causing the above food processing and production problems, such as small production scale, backward technique, insufficient supervision force of administrative supervision department, poor technology research and development and laggard detecting techniques. Even if raw materials of food processing are primary agricultural products and "scientization" or "ecologicalization" can be reached in animal feeding, "clenbuterol" event still exists. Many consumers cannot stand market allure and eat inferior products. This is the root source of food safety accidents. 


\section{Countermeasures and suggestions on food quality and safety control in production processing link}

Based on the above discussion, we can know food quality is closely related to our life and concerns our life health and safety. In current food safety accidents, people should continuously absorb experience, summarize the methods to improve food quality and safety and enhance attention to food hygiene, quality and safety. Government sector, food processing and production enterprises and other social departments should join hands to solve food safety problems, enhance sense of responsibility and take the initiative to undertake quality control and management responsibility in food processing.

Suggestions on enterprises. Firstly, enterprises should continuously enhance quality awareness. Weak quality awareness is a major cause for quality problem in food production and processing, and enterprises cannot strengthen quality supervision and management of production link. Thus, enterprise managers should establish a set of sound management system and let production and processing departments at each kevel strictly implement the system. Meanwhile, it is required to train and educate production personnel, improve their sense of responsibility and enhance quality management awareness. Secondly, enterprises should actively introduce advanced production experience, technology and facilities. This is the key to improving food processing efficiency and quality. Many food producing and production enterprises in China have obsolete manufacturing techniques, lag in technology and low production efficiency. Thus, enterprises should enhance communication and connection with each industry and each field, refer to technology and process of advanced production unit, increase technology research and development investment and boost market competitiveness by technology and process. At the same time, enterprises should intensify training of technical personnel, organize personnel to participate in technical seminar and industry exchange meeting at a regular interval, master development dynamics in the industry and acquire the latest technological achievements ${ }^{[3]}$.

Finally, thorough inspection system should be established. Food safety problems can be found in time only through inspection. After completing every batch of products, enterprises should carry out product quality inspection by thorough quality inspection procedure and system, establish and perfect test system, continuously standardize self-inspection behavior, debug detecting instruments, ensure accurate food detection results and make food inspection report more real and effective. If enterprises are incapable of self-inspection, they may entrust quality inspection institution for testing. In addition, original data of outgoing products and inspection report records files should be established. After product inspection meet criteria, they can be packed and sold.

Suggestions on government sector. Government sector is a third party between enterprises and consumers and independent of the two organizations. Government sector plays an important role in product quality information transfer and information asymmetry reduction. Food quality and safety management and control can be enhanced from the following aspects:

Firstly, laws and regulations should be perfected continuously. It is required to establish thorough supervision mechanism, make sure enterprises or individual workshops own standard guidance in food processing, keep these departments under strict supervision, standardize follow-up supervision mechanism of certified enterprises or production workshops and make enterprise production and processing activities always under standard state. Secondly, institutional deterrent effect should be strengthened. The government as a state organ has the right to manage food production and processing enterprises. Strict punishment system with strong binding effect can caution food production and processing enterprises. Media may be applied to expose food quality and safety accidents so that the whole society will pay attention to them. This will certainly caution enterprises. In this way, enterprises can absorb lessons while they are punished, and improve attention to food safety. For the enterprises with serious violation, their production license may be revoked, and they may be eliminated from food production market to standardize production and operation behaviors of other production enterprises. Award and punishment are also accompanied. It is necessary to actively praise and reward the enterprises with good product quality, high market reputation and remarkable contributions to food processing and production, encourage them to keep on optimizing and 
improving production mode and to become industry leaders and promote healthy and orderly development of the whole industry ${ }^{[4]}$.

Technical capacity construction should be strengthened. It is required to enhance exchange and cooperation among supervision departments at each level, construct uniform standard system and make products own quality standard and authority. Enterprises should be encouraged to participate in international food quality and safety management activities, and learning and research of foreign technological achievements should be enhanced. International production standards may be directly utilized or used for reference. For those which do not comply with development situations, self-dependent innovation should be conducted to create technology or process really suitable for production and processing. Meanwhile, it is required to focus on the latest development dynamics of domestic and foreign food detection technology, actively learn and refer to advanced detection technology, properly introduce and propose advanced detection technology, establish detection personnel training system, overall boost food detection ability and efficiency and improve supervision ability of supervision departments.

Risk management mechanism should be established. Government sector should regularly organize analysis and evaluation of food quality and safety risks, apply risk analysis technology in modification of laws and regulations, make law and regulation construction accord with norms through risk analysis, gather food quality risk information, establish quality risk management mechanism with food quality risk disposal being the center, continuously optimize risk management means, set up early warning mechanism of food quality and safety risks, collect information and data influencing quality safety and establish more smooth exchange mechanism. Finally, the supervision function of media and consumers should be fully exerted ${ }^{[5]}$. Quality problem exists in food processing. On the one hand, enterprises and relevant government departments supervise poorly; on the other hand, consumers and media are in duty bound to participate in food processing and production supervision. News media should actively cooperate and communicate with enterprises and government, report truthfully quality safety accidents in food processing and guide positive social opinion trend. Besides, the persons in charge of safety accidents should make a rational explanation for the public and consumers.

\section{Conclusions}

This paper mainly analyzes development status and problems of food processing industry in China, and proposes several hygiene and quality control methods in food processing in allusion to these problems. Enterprises, government regulation departments, news media and consumers should jointly participate in quality control and management in food processing and facilitate stable and sustainable development of Chinese food processing and production industry.

\section{References}

[1] Liu Changm Zhang Hao, An Yufa et al., Study on Weak Links, Root Reasons and Key Control Pintos of Chinese Food Quality and Safety - Empirical Analysis Based on 1460 Food Quality and Safety Accidents. Issues in Agricultural Economy, 2011(1):24-31.

[2] Zhang Hongxia, An Yufa, Theoretical and Empirical Analysis of Food Quality and Safety Signal Transmission. Research on Economics and Management, 2014(6):123-128.

[3] Zhong Zhen, Lei Fengshan, Liu Tongshan et al., General Frame Construction of Quality Economics - Basic Connotation of Food Quality and Safety. Soft Science, 2013,27(1):69-73.

[4] Ma Hongyan, Study on Weak Links and Key Control Points of Food Quality Safety - Analysis Based on Food Quality Security Accidents in Henan Province. Contemporary Economics, 2015(3):48-49. 
[5] Wang Huimin, Qiao Juan, Behavior and Benefit of Peasant Households Participating in Food Quality and Safety Traceability System - Case Study of Beijing Vegetable Cultivation Peasants. Issues in Agricultural Economy, 2011(2):45-51. 